\begin{tabular}{lll}
\hline VOL. $84 / 85$ & PART 1 \\
\hline
\end{tabular}

\title{
LARGE DEVIATIONS FOR GENERIC STATIONARY PROCESSES
}

BY

EMMANUEL LESIGNE (TOURS) AND DALIBOR VOLNÝ (ROUEN)

\begin{abstract}
Let $(\Omega, \mathcal{A}, \mu, T)$ be a measure preserving dynamical system. The speed of convergence in probability in the ergodic theorem for a generic function on $\Omega$ is arbitrarily slow.
\end{abstract}

Introduction. Let $(\Omega, \mathcal{A}, \mu)$ be a probability space and $T$ a measurable and measure preserving transformation of $\Omega$ onto itself. This dynamical system is supposed to be ergodic and aperiodic. For $p \in[1,+\infty]$, we denote by $L_{0}^{p}(\mu)$ the space of measurable functions with finite $p$ th moment and zero mean. If $f$ is a measurable function on $\Omega,\left(f \circ T^{i}\right)$ is a strictly stationary sequence of random variables. If $n$ is a positive integer, we define $S_{n}(f):=$ $\sum_{i=0}^{n-1} f \circ T^{i}$.

For $f \in L_{0}^{p}(\mu)$, the Ergodic Theorem asserts that the sequence of averages $\left(\frac{1}{n} S_{n}(f)\right)$ converges to zero in $L^{p}$ and almost surely. For the Central Limit Theorem, the strict stationarity is not a sufficient assumption. However, in any (aperiodic and ergodic) dynamical system there exists a dense set of $f \in L_{0}^{p}$ for which the Central Limit Theorem, the weak (Donsker) and the strong (Strassen) Invariance Principles, and the Law of Iterated Logarithm hold (cf. [V2], [Bu-Den], [L1], [L2]).

U. Krengel $([\mathrm{Kr}])$ showed that the convergence in the Ergodic Theorem can be arbitrarily slow and A. del Junco with J. Rosenblatt ([dJ-Ro]) showed that this event is generic. In [V1], D. Volný showed that for any admissible sequence $a_{n} \rightarrow \infty$ there is a generic set of $f \in L_{0}^{p}$ such that the distributions of $\left(1 / a_{n}\right) S_{n}(f)$ converge along subsequences to all probability laws.

The Ergodic Theorem implies

$$
\lim _{n \rightarrow \infty} \mu\left(S_{n}(f)>n\right)=0 .
$$

In this paper we study the speed of this convergence for dense and generic classes of functions in various spaces.

Let us notice that estimations of probabilities of large deviations for sums of independent random variables, for martingales or under mixing

2000 Mathematics Subject Classification: 60F10, 60G10, 28 D05. 
conditions are, since Cramér's work $([\mathrm{C}])$, a widely studied subject. The books by Petrov $([\mathrm{Pe}])$ and Dembo-Zeitouni $([\mathrm{Dem}-\mathrm{Z}])$ give a panorama of this subject.

We prove that, for an arbitrary sequence of real numbers $\left(b_{n}\right)$ going to infinity, for any $1 \leq p<\infty$ there exists a dense $G_{\delta}$ set of $f \in L_{0}^{p}(\mu)$ such that $\limsup _{n \rightarrow \infty}\left(b_{n} \mu\left(S_{n}(f)>n\right)\right)=\infty$ and there exists a dense set of $f \in L_{0}^{p}(\mu)$ such that $\liminf _{n \rightarrow \infty}\left(b_{n} \mu\left(S_{n}(f)>n\right)\right)=\infty$. Corresponding results are shown for spaces of bounded functions.

Let us recall a well known result.

THEOREM 1. Let $\left(c_{n}\right)_{n>0}$ be a sequence of real numbers with $c_{n} \rightarrow 0$. Then for every $1 \leq p \leq \infty$ there exists a dense $G_{\delta}$ set $G \subset L_{0}^{p}$ such that for every $f \in G$,

$$
\limsup _{n \rightarrow \infty} \mu\left(\left|S_{n}(f)\right|>n c_{n}\right)=1 .
$$

This theorem can be proved by the following argument: denote by $H_{n}$ the set of functions $f$ in $L_{0}^{p}$ for which there exists $k>n$ such that $\mu\left(\left|S_{k}(f)\right|>\right.$ $\left.k c_{k}\right)>1-1 / n$; this set is open in $L_{0}^{p}$, and by using the Rokhlin lemma, it can be shown to be dense; the intersection of these sets is a dense $G_{\delta}$ with the stated property.

\section{Generic properties}

THEOREM 2. Let $\left(a_{n}\right)$ be a sequence of positive numbers with $\sum a_{n}=\infty$. Let $\varrho$ be a strictly positive continuous increasing function on 10,1$]$. For every $1 \leq p<\infty$ there exists a dense $G_{\delta}$ set $G \subset L_{0}^{p}$ such that for every $f \in G$,

$$
\sum_{n=1}^{\infty} a_{n} \varrho\left(\mu\left(S_{n}(f)>n\right)\right)=\infty .
$$

This result implies

THEOREM 3. Let $\left(b_{n}\right)_{n>0}$ be a sequence of positive numbers with $b_{n} \rightarrow \infty$. For every $1 \leq p<\infty$ there exists a dense $G_{\delta}$ set $G \subset L_{0}^{p}$ such that for every $f \in G$,

$$
\limsup _{n \rightarrow \infty} b_{n} \mu\left(S_{n}(f)>n\right)=\infty .
$$

Proof. If $b_{n} \rightarrow \infty$, there exists an increasing function $\varrho$ satisfying the hypotheses of Theorem 2 and such that

$$
\sum_{n=1}^{\infty} \varrho\left(\frac{1}{\sqrt{b_{n}}}\right)<\infty .
$$

We choose $a_{n}=1, n=1,2, \ldots$ By Theorem 2 there exists a dense $G_{\delta}$ set 
$G \subset L_{0}^{p}$ such that for every $f \in G$,

$$
\sum_{n=1}^{\infty} \varrho\left(\mu\left(S_{n}(f)>n\right)\right)=\infty
$$

If $f \in G$ then, infinitely often, $\mu\left(S_{n}(f)>n\right)>1 / \sqrt{b_{n}}$.

Lemma 4. For any $\varepsilon \in] 0,1 / 4\left[\right.$ and any integer $k>0$ there exists $h \in L_{0}^{\infty}$ such that

$$
\mu\left(S_{j}(h) \geq 2 j: 1 \leq j \leq k\right) \geq \varepsilon \quad \text { and } \quad \int|h|^{p} d \mu \leq 2^{p+2} \varepsilon .
$$

Pr o o f. We consider a Rokhlin tower $A, T^{-1} A, \ldots, T^{-2 k+1} A$ and a measurable subset $B$ disjoint from the tower such that $\mu(A)=\varepsilon / k$ and $\mu(B)=$ $2 \varepsilon$. We consider the function $h$ equal to 2 on the tower, to -2 on $B$ and 0 everywhere else. The event $\left\{S_{j}(h) \geq 2 j: 1 \leq j \leq k\right\}$ contains the union of the $T^{-j} A$ for $k \leq j<2 k$.

Proof of Theorem 2. There exists a sequence $\left(\varepsilon_{n}\right)$ of positive numbers such that $\varepsilon_{n} \rightarrow 0$ and $\sum_{n=1}^{\infty} a_{n} \varepsilon_{n}=\infty$. We denote by $H_{n}$ the set of all $f \in L_{0}^{p}$ for which there exists a $k>n$ such that

$$
\sum_{j=1}^{k} a_{j} \varrho\left(\mu\left(S_{j}(f)>j\right)\right)>\sum_{j=1}^{k} a_{j} \varepsilon_{j} .
$$

Each $H_{n}$ is open in $L_{0}^{p}$. We shall prove that it is dense. The intersection of all the $H_{n}$ is the set $G$ with the property described in the theorem.

The functions $g-g \circ T$ with $g \in L^{\infty}$ form a dense subset of $L_{0}^{p}$ (cf. [Pa, p. 23]). We now show that $H_{n}$ intersects each neighbourhood of any of them.

We consider an integer $n$, a bounded measurable function $g$ and a positive $\varepsilon<1 / 4$. We fix an integer $l>2\|g\|_{\infty}$ such that

$$
j>l \Rightarrow \varepsilon_{j}<\frac{1}{2} \varrho(\varepsilon)
$$

and an integer $k>n, l$ such that

$$
\sum_{j=1}^{l} a_{j} \varepsilon_{j}<\sum_{j=l+1}^{k} a_{j} \varepsilon_{j} .
$$

By Lemma 4 there exists a function $h$ such that

$$
\int|h|^{p} d \mu \leq 2^{p+2} \varepsilon \quad \text { and } \quad \mu\left(S_{j}(h) \geq 2 j\right) \geq \varepsilon \quad \text { for all } 1 \leq j \leq k .
$$

Let $f=h+g-g \circ T$. For $j>l$ we have

$$
\varrho\left(\mu\left(S_{j}(f) \geq j\right)\right) \geq \varrho\left(\mu\left(S_{j}(h) \geq 2 j\right)\right) \geq \varrho(\varepsilon) .
$$


Therefore

$$
\sum_{j=1}^{k} a_{j} \varrho\left(\mu\left(S_{j}(f) \geq j\right)\right) \geq \sum_{j=l+1}^{k} a_{j} \varrho(\varepsilon)>2 \sum_{j=l+1}^{k} a_{j} \varepsilon_{j}>\sum_{j=1}^{k} a_{j} \varepsilon_{j} .
$$

Thus $f \in H_{n}$ and $\|f-(g-g \circ T)\|_{p}=\|h\|_{p} \leq\left(2^{p+2} \varepsilon\right)^{1 / p}$.

It is easy to observe that Theorem 3 cannot be extended to $L_{0}^{\infty}$. However, the next two theorems give results of the same type for bounded functions.

REMARK. In the proof of Theorem 2 we used the fact that the coboundaries $g-g \circ T$ with $g \in L^{\infty}$ form a dense subset in $L_{0}^{p}, 1 \leq p<\infty$. This, however, is not true for $p=\infty$ : in any ergodic and aperiodic dynamical system and for each $p>0$ there exist $f \in L_{0}^{p}$ and $\varepsilon>0$ such that for each coboundary $g-g \circ T \in L_{0}^{\infty}$ with $g \in L^{p}$ we have $\|f-(g-g \circ T)\|_{\infty}>\varepsilon$ (cf., e.g., $[\mathrm{K}]$ or $[\mathrm{V}-\mathrm{W}]$ ). In $[\mathrm{V}-\mathrm{W}]$ it is shown that for each $1 \leq p<\infty$, the closure (in $L_{0}^{\infty}$ ) of the set of all $g-g \circ T \in L_{0}^{\infty}, g \in L^{p}$, can be characterized by the rate of decay of the probabilities of $\mu\left(\left|S_{n}(f)\right|>n c\right)$.

Let $\mathcal{B}$ be the set of real measurable functions $f$ bounded by one and of zero mean, endowed with the distance

$$
d(f, g)=\inf \{a>0: \mu(|f-g|>a)<a\} .
$$

The metric space $(\mathcal{B}, d)$ is complete. Let $\mathcal{M}$ be the set of elements of $\mathcal{B}$ taking only the values -1 and 1 . This set is closed in $(\mathcal{B}, d)$.

THEOREM 5. Let $\left(b_{n}\right)_{n>0}$ be a sequence of positive numbers with $b_{n} \rightarrow \infty$. For every $c<1$, in the space $(\mathcal{B}, d)$ there is a dense $G_{\delta}$ set of functions $f$ satisfying

$$
\limsup _{n \rightarrow \infty} b_{n} \mu\left(\left|S_{n}(f)\right|>c n\right)=\infty .
$$

In the space $(\mathcal{M}, d)$ there is a dense $G_{\delta}$ set of functions $f$ satisfying

$$
\limsup _{n \rightarrow \infty} b_{n} \mu\left(\left|S_{n}(f)\right|=n\right)=\infty .
$$

The proof of Theorem 5 is similar to the one of Theorem 1 (see also [V1]) and is left to the reader.

THEOREM 6. Let $\left(b_{n}\right)_{n>0}$ be a sequence of positive numbers with $b_{n} \rightarrow \infty$. There exists a dense $G_{\sigma \delta}$ subset $G \subset L_{0}^{\infty}$ such that for every $f \in G$,

$$
\limsup _{n \rightarrow \infty} b_{n} \mu\left(\left|S_{n}(f)\right|>c n\right)=\infty
$$

holds for some $c>0$.

Proof. For $c>0$ define

$$
\begin{aligned}
V_{c} & =\left\{f: \limsup _{n \rightarrow \infty} b_{n} \mu\left(\left|S_{n}(f)\right|>c n\right)=\infty\right\}, \\
V_{c, n} & =\left\{f: \exists k \geq n, b_{k} \mu\left(\left|S_{k}(f)\right|>c k\right)>n\right\} .
\end{aligned}
$$


We have $V_{c}=\bigcap_{n=1}^{\infty} V_{c, n}$, and every $f \in \bigcup_{l=1}^{\infty} V_{1 / l}$ satisfies (1). Each $V_{c, n}$ is open in $L_{0}^{\infty}$. Therefore $\bigcup_{l=1}^{\infty} V_{1 / l}$ is $G_{\sigma \delta}$.

Let $f \in L_{0}^{\infty}$ and $\varepsilon>0$. Theorem 5 guarantees the existence of a function $g$ in $V_{2 \varepsilon}$ bounded by $3 \varepsilon$. If $f \notin V_{\varepsilon}$, we have $f+g \in V_{\varepsilon}$. This proves that $V_{\varepsilon}$ is $3 \varepsilon$-dense in $L_{0}^{\infty}$. Therefore $\bigcup_{l=1}^{\infty} V_{1 / l}$ is dense.

Properties of a dense class. We now present a "lim inf" type result.

THEOREM 7. Let $\left(b_{n}\right)_{n>0}$ be a sequence of real numbers with $b_{n} \rightarrow \infty$. There is a bounded measurable function $f$ on $\Omega$ such that $\int f d \mu=0$ and

$$
\liminf _{n \rightarrow \infty} b_{n} \mu\left(S_{n}(f) \geq n\right)=\infty .
$$

Remarks. 1 . The set of functions $f$ with the above property is in fact dense in every $L_{0}^{p}(\mu), 1 \leq p<\infty$.

2. However, in each $L_{0}^{p}(\mu)$, there is a dense $G_{\delta}$ set of functions $f$ with

$$
\liminf _{n \rightarrow \infty} b_{n} \mu\left(S_{n}(f) \geq n\right)=0 .
$$

Both statements follow from the fact that the set of coboundaries $g-g \circ T$ with $g$ measurable and bounded is dense in each $L_{0}^{p}, 1 \leq p<\infty$. In the proof of the second remark we need a Baire argument as in the proof of Theorem 1 or Theorem 2.

In the next lemma and in the proof of Theorem 7 , we call an element $A$ of $\mathcal{A}$ the base of a Rokhlin tower of height $n$ if $A, T A, \ldots, T^{n-1} A$ are measurable, pairwise disjoint and have the same measure. The family $A, T A, \ldots, T^{n-1} A$ is called a Rokhlin tower. Even if $T$ is not invertible, we can construct such a tower: it suffices, using the Rokhlin Lemma, to take a tower $C, T^{-1} C, \ldots, T^{-n+1} C$ and define $A=T^{-n+1} C$.

Lemma 8. Let $n<m$ be positive integers. Let $A, T A, \ldots, T^{4 n-1} A$ and $B, T B, \ldots, T^{2 m-1} B$ be two Rokhlin towers. Put $\bar{A}=A \cup T A \cup \ldots \cup T^{4 n-1} A$ and $\bar{B}=B \cup T B \cup \ldots \cup T^{2 m-1} B$. There exists $A^{\prime} \subset A$ such that

$$
\frac{\mu\left(A^{\prime}\right)}{\mu(A)} \leq 4 \frac{\mu(\bar{B})}{\mu(\bar{A})}
$$

and, for each $n \leq k<3 n$,

$$
\bar{B} \cap T^{k} A \subset T^{k} A^{\prime} .
$$

Lemma 8 says that the intersection of the tower $\bar{B}$ with the $2 n$ middle floors of the tower $\bar{A}$ is contained in a subtower of $\bar{A}$ of measure smaller than $4 \mu(\bar{B})$. Here, by a "subtower of $\bar{A}$ ", we mean a tower $A^{\prime}, T A^{\prime}, \ldots, T^{4 n-1} A^{\prime}$ with $A^{\prime} \subset A$. A proof of this lemma is given at the end of the paper. 
Proof of Theorem 7. Let $\left(n_{k}\right)_{k \geq 1}$ be an increasing sequence of positive integers such that

$$
b_{n_{1}}>4, \quad 100 b_{n_{k}}<b_{n_{k+1}} \text { and } \quad b_{n_{k}}=\inf _{n \geq n_{k}} b_{n} .
$$

With each $k$ we associate a Rokhlin tower $A_{k}, T A_{k}, \ldots, T^{8 n_{k+1}-1} A_{k}$ such that $\mu\left(\bar{A}_{k}\right)=1 / \sqrt{b_{n_{k}}}$ and a set $B_{k}$ in $\mathcal{A}$ disjoint from $\bigcup_{j=1}^{k} \bar{A}_{j}$ such that $\mu\left(B_{k}\right)=1 / \sqrt{b_{n_{k}}}$. (As before, we use the notation $\bar{A}_{k}=\bigcup_{0 \leq j<8 n_{k+1}} T^{j} A_{k}$ ).

We recursively define a sequence $\left(f_{k}\right)$ of functions by

$$
f_{1}= \begin{cases}1 & \text { on } \bigcup_{j=0}^{4 n_{2}-1} T^{j} A_{1}, \\ -1 & \text { on } \bigcup_{j=4 n_{2}}^{8 n_{2}-1} T^{j} A_{1}, \\ 0 & \text { otherwise, }\end{cases}
$$

and

$$
f_{k}= \begin{cases}1 & \text { on } \bigcup_{j=0}^{4 n_{k+1}-1} T^{j} A_{k}, \\ -1 & \text { on } \bigcup_{j=4 n_{k+1}}^{8 n_{k+1}-1} T^{j} A_{k}, \\ f_{k-1} & \text { on }\left(\bar{A}_{k} \cup B_{k}\right)^{\mathrm{c}}, \\ c_{k} & \text { on } B_{k},\end{cases}
$$

where the constant value $c_{k}$ of the function $f_{k}$ on $B_{k}$ is chosen so that the mean value of $f_{k}$ on $\Omega$ is zero. Because $\mu\left(f_{k} \neq f_{k-1}\right) \leq 2 / \sqrt{b_{n_{k}}}$ is a summable sequence, the sequence $\left(f_{k}\right)$ converges almost surely to a function $f$. Notice that all these functions are bounded by one.

By construction (recall that $B_{l} \cap \bar{A}_{k}=\emptyset$ for $l>k$ ), we have

$$
\left(\left\{f \neq f_{k}\right\} \cap \bar{A}_{k}\right) \subset \bigcup_{l>k} \bar{A}_{l} .
$$

Let $k<l$. By Lemma 8 we know that the trace of the tower $\bar{A}_{l}$ on the $4 n_{k+1}$ middle floors of the tower $\bar{A}_{k}$ is contained in a subtower of $\bar{A}_{k}$ of measure smaller than $4 \mu\left(\bar{A}_{l}\right)$. This implies that the set

$$
\left\{f \neq f_{k}\right\} \cap \bigcup_{j=2 n_{k+1}}^{6 n_{k+1}-1} T^{j} A_{k}
$$

is contained in a subtower $\bar{A}_{k}^{\prime}$ of $\bar{A}_{k}$ such that

$$
\mu\left(\bar{A}_{k}^{\prime}\right) \leq 4 \sum_{l>k} \mu\left(\bar{A}_{l}\right)
$$

We have

$$
\mu\left(\bar{A}_{k}^{\prime}\right) \leq 4 \sum_{l>k} 10^{k-l} \mu\left(\bar{A}_{k}\right)<\frac{1}{2} \mu\left(\bar{A}_{k}\right),
$$

which implies $\mu\left(A_{k}^{\prime}\right)<\frac{1}{2} \mu\left(A_{k}\right)$. 
Let $n$ be a positive integer and $k$ be such that $n_{k} \leq n<n_{k+1}$. We have $S_{n}\left(f_{k}\right)=n$ on the set $\bigcup_{j=0}^{4 n_{k+1}-n-1} T^{j} A_{k}$. On the set $\bigcup_{j=2 n_{k+1}}^{3 n_{k+1}-1} T^{j}\left(A_{k} \backslash A_{k}^{\prime}\right)$, which is included in the preceding one, we have $S_{n}(f)=S_{n}\left(f_{k}\right)=n$. Thus

$$
\mu\left(S_{n}(f)=n\right) \geq n_{k+1} \mu\left(A_{k} \backslash A_{k}^{\prime}\right) \geq \frac{1}{2} n_{k+1} \mu\left(A_{k}\right)=\frac{1}{16 \sqrt{b_{n_{k}}}} \geq \frac{1}{16 \sqrt{b_{n}}},
$$

which proves the theorem.

Proof of Lemma 8. We give a detailed proof in the case where the measure preserving transformation $T$ is invertible. The argument can be extended to the non-invertible case without difficulty if there exist $C$ and $D$ in $\mathcal{A}$ such that $A=T^{-4 n+1} C$ and $B=T^{-2 m+1} D$.

Define

$$
A^{\prime}=\bigcup_{k=n}^{3 n-1}\left(A \cap T^{-k} \bar{B}\right) .
$$

In the tower $A, T A, \ldots, T^{4 n-1} A$, above each point $\omega \in A^{\prime}$ there are at least $n$ points $T^{k} \omega \in \bar{B}$. Indeed, if $\omega \in A^{\prime}$, there exists $k$ between $n$ and $3 n-1$ such that $T^{k} \omega \in \bar{B}$. Then either $T^{k+j} \omega \in \bar{A} \cap \bar{B}$ for each $0 \leq j \leq n$ or $T^{k-j} \omega \in \bar{A} \cap \bar{B}$ for each $0 \leq j \leq n$. (Here we used two facts: if $\omega^{\prime} \in \bar{B}$, then $T^{j} \omega^{\prime} \in \bar{B}$ either for any $0 \leq j \leq n$ or for any $0 \geq j \geq-n$; and if $\omega^{\prime} \in T^{k} A$ for one $k$ between $n$ and $3 n-1$, then $T^{j} \omega^{\prime} \in \bar{A}$ for any $j$ between $n$ and $-n$.)

We claim that this implies

$$
n \mu\left(A^{\prime}\right) \leq \mu(\bar{B}) .
$$

Indeed, for each subset $K$ of $E=\{0,1, \ldots, 4 n-1\}$, define

$$
A_{K}^{\prime}=\left\{\omega \in A^{\prime}: T^{k}(\omega) \in \bar{B} \text { for } k \in K \text { and } T^{k}(\omega) \notin \bar{B} \text { for } k \in E \backslash K\right\} .
$$

The sets $A_{K}^{\prime}$ form a partition of $A^{\prime}$. We have $A_{K}^{\prime}=\emptyset$ if $\operatorname{card}(K)<n$. The sets $T^{k} A_{K}^{\prime}$, for $K \subset E$ and $k \in K$, are pairwise disjoint and included in $\bar{B}$. Thus

$$
\mu(\bar{B}) \geq \sum_{K \subset E} \sum_{k \in K} \mu\left(T^{k} A_{K}^{\prime}\right) \geq n \sum_{K \subset E} \mu\left(A_{K}^{\prime}\right)=n \mu\left(A^{\prime}\right) .
$$

\section{REFERENCES}

[Bu-Den] R. Burton and M. Denker, On the central limit theorem for dynamical systems, Trans. Amer. Math. Soc. 302 (1987), 715-726.

[C] H. Cramér, Sur un nouveau théorème-limite de la théorie des probabilités, in: Actualités Sci. Indust. 736, Hermann, Paris, 1938, 5-23.

[Dem-Z] A. Dembo and O. Zeitouni, Large Deviations Techniques and Applications, Jones and Bartlett, Boston, 1993 (or: Appl. Math. 38, Springer, 1998). 
[dJ-Ro] A. del Junco and J. Rosenblatt, Counterexamples in ergodic theory and in number theory, Math. Ann. 245 (1979), 185-197.

$[\mathrm{K}] \quad$ A. Katok, Constructions in ergodic theory, unpublished manuscript.

$[\mathrm{Kr}] \mathrm{U} . \mathrm{Krengel,}$ On the speed of convergence in the ergodic theorem, Monatsh. Math. 86 (1978), 3-6.

[L1] M. Lacey, On weak convergence in dynamical systems to self-similar processes with spectral representation, Trans. Amer. Math. Soc. 328 (1991), 767-778.

[L2] - On central limit theorems, modulus of continuity and Diophantine type for irrational rotations, J. Anal. Math. 61 (1993), 47-59.

[Pa] W. Parry, Topics in Ergodic Theory, Cambridge Univ. Press, 1981.

[Pe] V. V. Petrov, Limit Theorems of Probability Theory, Oxford Stud. Probab. 4, Oxford Sci. Publ., Oxford Univ. Press, 1995.

[V1] D. Volný, On limit theorems and category for dynamical systems, Yokohama Math. J. 38 (1990), 29-35.

[V2] - Invariance principles and Gaussian approximation for strictly stationary processes, Trans. Amer. Math. Soc. 351 (1999), 3351-3371.

[V-W] D. Volný and B. Weiss, Coboundaries in $L_{0}^{\infty}$, in preparation.

Laboratoire de Mathématiques

et Physique Théorique

UPRES-A 6083 CNRS

Université François Rabelais

Parc de Grandmont

F-37200 Tours, France

E-mail: lesigne@univ-tours.fr
Laboratoire d'Analyse et Modèles Stochastiques UPRES-A 6085 CNRS Université de Rouen F-76821 Mont-Saint-Aignan Cedex, France E-mail: dalibor.volny@univ-rouen.fr 